\title{
TWISTINGS, CROSSED COPRODUCTS, AND HOPF-GALOIS COEXTENSIONS
}

\section{S. CAENEPEEL, DINGGUO WANG, and YANXIN WANG}

\author{
Received 1 December 2002
}

\begin{abstract}
Let $H$ be a Hopf algebra. Ju and Cai (2000) introduced the notion of twisting of an $H$-module coalgebra. In this paper, we study the relationship between twistings, crossed coproducts, and Hopf-Galois coextensions. In particular, we show that a twisting of an $H$-Galois coextension remains $H$-Galois if the twisting is invertible.
\end{abstract}

2000 Mathematics Subject Classification: 16W30.

1. Introduction. A fundamental result in Hopf-Galois theory is the normal basis theorem, stating that, for a finitely generated cocommutative Hopf algebra $H$ over a commutative ring $k$, the set of isomorphism classes of Galois $H$-objects that are isomorphic to $H$ as an $H$-comodule is a group, and this group is isomorphic to the second Sweedler cohomology group $H^{2}(H, k)$ (see [14]). The Galois object corresponding to a 2-cocycle is then given by a crossed product construction. The crossed product construction can be generalized to arbitrary Hopf algebras and plays a fundamental role in the theory of extensions of Hopf algebras (see [3, 11]). Also in this more general situation, it turns out that there is a close relationship between crossed products on one side and Hopf-Galois extensions and cleft extensions on the other side (cf. [3, 4, 11]). A survey can be found in [13]. An alternative way to deform the multiplication on an $H$-comodule algebra $A$ has been proposed in [1], using the so-called twisting of $A$, and it was shown that the crossed product construction can be viewed as a special case of the twisting construction. The relation between twistings and $H$-Galois extensions was studied in [2].

Now, there exists a coalgebra version of the normal basis theorem (see [6]). In this situation, one tries to deform the comultiplication on a commutative Hopf algebra $H$, using this time a Harrison cocycle instead of a Sweedler cocycle. Crossed coproducts, cleft coextensions, and Hopf-Galois coextensions have been introduced and studied in [8, 10]. Ju and Cai [12] have introduced the notion of twisting of an $\mathrm{H}$-module coalgebra, which can be viewed as a dual version of the twistings introduced in [1]. The aim of this paper is to study the relationship between twistings, crossed coproducts, and Hopf-Galois coextensions. Our main result is the fact that the twisting of a Hopf-Galois coextension by an invertible twist map is again a Hopf-Galois coextension (and vice versa). 
Our paper is set up as follows. In Section 2.1, we recall the twistings introduced in [12], and in Section 2.2 we recall the definition of a Harrison cocycle and the crossed coproduct construction from [8, 10]. In Section 3, we introduce an alternative version of 2-cocycles, called twisted 2-cocycles, and discuss the relation with Harrison cocycles (Proposition 3.3). In Section 4, we introduce an equivalence relation on the set of twistings of an $H$-module coalgebra and we show that a twisting in an equivalence class is invertible if and only if all the other twistings in this equivalence class are invertible (Theorem 4.4). Two twistings are equivalent if and only if their corresponding crossed coproducts are isomorphic (Proposition 2.1). In Section 5, the relationship between twistings and Hopf-Galois coextensions is investigated.

For the general theory of Hopf algebras, we refer to the literature (see, e.g, $[9,13,15])$.

2. Notation and preliminary results. We work over a field $k$. All maps are assumed to be $k$-linear. For the comultiplication on a $k$-coalgebra $C$, we use the Sweedler-Heyneman notation

$$
\Delta_{C}(c)=c_{1} \otimes c_{2}
$$

with the summation implicitly understood. We use a similar notation for a (right) coaction of a coalgebra on a comodule:

$$
\rho(m)=m_{0} \otimes m_{1} \in M \otimes C .
$$

Let $A$ be a $k$-algebra, then $\operatorname{Hom}(C, A)$ is also an algebra, with convolution product

$$
(f * g)(c)=f\left(c_{1}\right) g\left(c_{2}\right) .
$$

We will denote by $\operatorname{Reg}(C, A)$ the set of convolution invertible elements in $\operatorname{Hom}(C, A)$, and $\mu_{A}^{C}$ will be the category of modules with a right $A$-action and a right $C$-coaction, such that the $C$-coaction is $A$-linear.

2.1. Twistings of a coalgebra. We recall some definitions and results from [12]. Let $H$ be a Hopf algebra over a field $k$, with bijective antipode $S$. The composition inverse of the antipode will be denoted by $\bar{S}$.

Recall that a right $H$-module coalgebra is a coalgebra $C$ which is also a right $H$-module such that

$$
\Delta(c \cdot h)=c_{1} \cdot h_{1} \otimes c_{2} \cdot h_{2}, \quad \varepsilon_{C}(c \cdot h)=\varepsilon_{C}(c) \varepsilon_{H}(h)
$$

for all $c \in C$ and $h \in H$. 
Consider the category $\mu_{H}^{C}$, whose objects are right $H$-modules and right $C$ comodules $M$ such that the following compatibility relation is satisfied:

$$
\rho(m \cdot h)=m_{0} \cdot h_{1} \otimes m_{1} \cdot h_{2}
$$

Recall from [12] that we have the following associative multiplication on $\operatorname{Hom}(C, H \otimes C)$ :

$$
\tau * \lambda=\left(m_{H} \otimes \operatorname{id}_{C}\right) \circ\left(\operatorname{id}_{H} \otimes \lambda\right) \circ \tau
$$

for all $\tau, \lambda \in \operatorname{Hom}(C, H \otimes C)$. The unit of this multiplication is the map $\sigma: C \rightarrow$ $H \otimes C, \sigma(c)=1 \otimes c$.

Remark that we have an algebra isomorphism

$$
\alpha: \operatorname{Hom}(C, H \otimes C) \longrightarrow{ }_{H} \operatorname{End}(H \otimes C)^{\mathrm{op}},
$$

where $\alpha$ is defined as follows. Take $\tau: C \rightarrow H \otimes C$, and write $\tau(c)=C_{-1} \otimes c_{0}$ (summation is understood). Then $\alpha(\tau)=f_{\tau}: H \otimes C \rightarrow H \otimes C$ is given by

$$
f_{\tau}(h \otimes c)=h \tau(c)=h c_{-1} \otimes c_{0}
$$

Assume that $\tau$ satisfies the following normality conditions:

$$
\left(1 \otimes \varepsilon_{C}\right) \tau(c)=\varepsilon(c) 1_{H}, \quad\left(\varepsilon_{H} \otimes 1\right) \tau(c)=c
$$

or

$$
c_{-1} \varepsilon_{C}\left(c_{0}\right)=\varepsilon_{C}(c) 1_{H}, \quad \varepsilon_{H}\left(c_{-1}\right) c_{0}=c .
$$

We can then define a new (in general noncoassociative) comultiplication $\Delta_{\tau}$ on $C$ as follows:

$$
\Delta_{\tau}(c)=c_{1} \cdot c_{2,-1} \otimes c_{2,0} \quad \text { or } \quad \Delta_{\tau}=\left(\psi_{H} \otimes \mathrm{id}\right) \circ(\mathrm{id} \otimes T) \circ \Delta,
$$

where $\psi_{H}: C \otimes H \rightarrow C$ is the right $H$-action on $C$, and where we used the Sweedler-type notation (id $\otimes T)(\Delta c)=c_{1} \otimes c_{2,-1} \otimes c_{2,0}$. Let $C^{\top}$ be equal to $C$ as a right $H$-module, with comultiplication $\Delta_{\tau}(c)$. A similar construction applies to $M \in \mathcal{M}_{H}^{C}: F_{\tau}(M)=M^{\top}$ as a right $H$-comodule, with

$$
\rho^{\top}(m)=m_{0} \cdot m_{1,-1} \otimes m_{1,0},
$$

where $\tau$ is called a twisting if and only if $C^{\top}$ is a right $H$-module coalgebra, and $M^{\tau} \in \mathcal{M}_{H}^{C^{\tau}}$ for all $M \in \mathcal{M}_{H}^{C}$. It is shown in [12, Theorem 1.1] that $\tau: C \rightarrow H \otimes C$, 
satisfying (2.9), is a twisting if and only if for all $h \in H$ and $c \in C$,

$$
\begin{gathered}
c_{-1} h_{1} \otimes c_{0} \cdot h_{2}=h_{1}\left(c \cdot h_{2}\right)_{-1} \otimes\left(c \cdot h_{2}\right)_{0}, \\
c_{-1} \otimes c_{0,1} \cdot c_{0,2,-1} \otimes c_{0,2,0}=c_{1,-1} c_{2,-1,1} \otimes c_{1,0} \cdot c_{2,-1,2} \otimes c_{2,0},
\end{gathered}
$$

where (2.13) is equivalent to

$$
S\left(h_{1}\right) c_{-1} h_{2} \otimes c_{0} \cdot h_{3}=(c \cdot h)_{-1} \otimes(c \cdot h)_{0}
$$

If $\tau$ has an inverse $\lambda$, then the functor $F$ is an equivalence of categories.

Left-hand twistings are defined in a similar way. Consider the vector space isomorphism

$$
\operatorname{Hom}(C, C \otimes H) \cong \operatorname{End}_{H}\left(C \otimes H^{\mathrm{op}}, C \otimes H^{\mathrm{op}}\right)
$$

The composition on the right-hand side is transported into the following associative multiplication on $\operatorname{Hom}(C, C \otimes H)$ :

$$
\tau \times \lambda=T \circ(T \circ \lambda * T \circ \tau) .
$$

Here, $T$ is the usual twist map. The unit $\sigma^{\prime}$ on $\operatorname{Hom}(C, C \otimes H)$ is given by $\sigma^{\prime}(c)=c \otimes 1$. If $\lambda \in \operatorname{Hom}(C, C \otimes H)$ satisfies the normalizing conditions

$$
\left(1 \otimes \varepsilon_{H}\right) \lambda(c)=c, \quad\left(\varepsilon_{C} \otimes 1\right) \lambda(c)=\varepsilon_{C}(c) 1_{H},
$$

then we can twist the comultiplication on $C$ as follows. Write $\lambda(c)=c_{0} \otimes c_{1}$, and define ${ }_{\lambda} \Delta$ by

$$
{ }_{\lambda} \Delta(c)=c_{1,0} \otimes c_{2} \cdot c_{1,1} .
$$

Let ${ }^{\lambda} C$ be equal to $C$ as a right $H$-module, equipped with the comultiplication ${ }_{\lambda} \Delta$. The $C$-coaction $M \in{ }^{C} \mathcal{M}_{H}$ can also be twisted as follows:

$$
{ }^{\lambda} \rho(m)=m_{-1,0} \otimes m_{0} m_{-1,1},
$$

where $\lambda$ is called a left-hand twisting if ${ }^{\lambda} C$ is an $H$-module coalgebra, and ${ }^{\lambda} M \in{ }^{\lambda} C_{M_{H}}$ for every $M \in{ }^{C} \mu_{H}$. The map $\lambda: C \rightarrow C \otimes H$ satisfying (2.18) is a left-hand twisting if and only if for all $h \in H$ and $c \in C$,

$$
\begin{gathered}
c_{0} \cdot h_{1} \otimes c_{1} h_{2}=\left(c \cdot h_{1}\right)_{0} \otimes h_{2}\left(c \cdot h_{1}\right)_{1}, \\
c_{0,1,0} \otimes c_{0,2} \cdot c_{0,1,1} \otimes c_{1}=c_{1,0} \otimes c_{2,0} \cdot c_{1,1,1} \otimes c_{2,1} c_{1,1,2} .
\end{gathered}
$$


Equation (2.21) is equivalent to

$$
c_{0} \cdot h_{1} \otimes \bar{S}\left(h_{3}\right) c_{1} h_{2}=\sum(c \cdot h)_{0} \otimes(c \cdot h)_{1} .
$$

For $\tau \in \operatorname{Hom}(C, H \otimes C)$ with inverse $\lambda$, we write

$$
\tau(c)=c_{-1} \otimes c_{0}, \quad \lambda(c)=c_{(-1)} \otimes c_{(0)} .
$$

We then have

$$
\mathcal{C}_{-1} \mathcal{C}_{0,(-1)} \otimes \mathcal{C}_{0,(0)}=\mathcal{C}_{(-1)} \mathcal{C}_{(0),-1} \otimes \mathcal{C}_{(0), 0}=1 \otimes \mathcal{C} .
$$

For $\gamma \in \operatorname{Hom}(C, C \otimes H)$ with inverse $\mu$, we write

$$
\gamma(c)=c_{0} \otimes c_{1}, \quad \mu(c)=c_{(0)} \otimes c_{(1)} .
$$

Let $\mathscr{T}(C)$ and $\mathscr{L}(C)$ be the sets of twistings and left-hand twistings of $C$, respectively, and $U(\mathscr{T}(C))$ and $U(\mathscr{L}(C))$ the sets of invertible twistings and left-hand twistings, respectively.

Proposition 2.1. Take $\tau \in U(\mathcal{T}(C))$ with inverse $\lambda$. Define $\ell(\tau): C \rightarrow C \otimes H$ by

$$
\ell(\tau)(c)=c_{0,(0)} \cdot \bar{S}\left(c_{0,(-1)}\right) \bar{S}\left(c_{-1}\right)_{1} \otimes \bar{S}\left(c_{-1}\right)_{2}
$$

Take $\gamma \in U(\mathscr{L}(C))$, with inverse $\mu$. Define $r(\gamma): C \rightarrow H \otimes C$ by

$$
r(\gamma)(c)=S\left(c_{1}\right)_{1} \otimes c_{0,(0)} \cdot S\left(c_{0,(1)}\right) S\left(c_{1}\right)_{2} .
$$

Then $\ell: U(\mathcal{T}(C)) \rightarrow U(\mathscr{L}(C))$ is a bijection with inverse $r$. Furthermore, $\ell(\sigma)=$ $\sigma^{\prime}$ and $r\left(\sigma^{\prime}\right)=\sigma$.

Proof. It is shown in [12] that $\ell(\tau) \in U(\mathscr{L}(C))$ with sn inverse given by

$$
\ell(\boldsymbol{\tau})^{\prime}(c)=c_{0,(0)} \cdot \bar{S}\left(c_{0,(-1)}\right)_{1} \bar{S}\left(c_{-1}\right)_{1} \otimes \bar{S}\left(\bar{S}\left(c_{-1}\right)_{3}\right) \bar{S}\left(c_{0,(-1)}\right)_{2} \bar{S}\left(c_{-1}\right)_{2}
$$

Set $g=\bar{S}\left(c_{0,(-1)}\right), h=\bar{S}\left(c_{-1}\right)$. Then $\ell(\tau)(c)=c_{0,(0)} \cdot g h_{1} \otimes h_{2}$, so 


$$
\begin{aligned}
r(\ell(\tau))(c)= & S\left(h_{2}\right)_{1} \otimes\left(c_{0,(0)} \cdot g h_{1}\right)_{0,(0)} \cdot \bar{S}\left(\left(c_{0,(0)} \cdot g h_{1}\right)_{0,(-1)}\right)_{1} \\
& \cdot \bar{S}\left(\left(c_{0,(0)} \cdot g h_{1}\right)_{-1}\right)_{1} S\left(\bar{S}\left(\bar{S}\left(\left(c_{0,(0)} \cdot g h_{1}\right)_{-1}\right)_{3}\right)\right. \\
& \left.\cdot \bar{S}\left(\left(c_{0,(0)} \cdot g h_{1}\right)_{0,(-1)}\right)_{2} \bar{S}\left(\left(c_{0,(0)} \cdot g h_{1}\right)_{-1}\right)_{2}\right) S\left(h_{2}\right)_{2} \\
= & S\left(h_{2}\right)_{1} \otimes\left(c_{0,(0)} \cdot g h_{1}\right)_{0,(0)} \bar{S}\left(\left(c_{0,(0)} \cdot g h_{1}\right)_{0,(-1)}\right)_{1} \\
& \cdot \bar{S}\left(\left(c_{0,(0)} \cdot g h_{1}\right)_{-1}\right)_{1} S\left(\bar{S}\left(\left(c_{0,(0)} \cdot g h_{1}\right)_{-1}\right)_{2}\right) \\
& \cdot S\left(\bar{S}\left(\left(c_{0,(0)} \cdot g h_{1}\right)_{0,(-1)}\right)_{2}\right) \bar{S}\left(\left(c_{0,(0)} \cdot g h_{1}\right)_{-1}\right)_{3} S\left(h_{2}\right)_{2} \\
= & S\left(h_{2}\right)_{1} \otimes\left(c_{0,(0)} \cdot g h_{1}\right)_{0,(0)} \\
& \cdot \varepsilon\left(\left(c_{0,(0)} \cdot g h_{1}\right)_{0,(-1)}\right) \bar{S}\left(\left(c_{0,(0)} \cdot g h_{1}\right)_{-1}\right) S\left(h_{2}\right)_{2} \\
= & S\left(h_{2}\right)_{1} \otimes\left(c_{0,(0)} \cdot g h_{1}\right)_{0} \cdot \bar{S}\left(\left(c_{0,(0)} \cdot g h_{1}\right)_{-1}\right) S\left(h_{2}\right)_{2} \\
= & S\left(h_{2}\right)_{1} \otimes c_{0,(0), 0} \cdot\left(g h_{1}\right)_{3} \bar{S}\left(\left(g h_{1}\right)_{2}\right) \bar{S}\left(c_{0,(0),-1}\right) \\
& \cdot \bar{S}\left(S\left(g h_{1}\right)_{1}\right) S\left(h_{2}\right)_{2} \\
= & S\left(h_{3}\right) \otimes c_{0,(0), 0} \cdot \bar{S}\left(c_{0,(0),-1}\right) g h_{1} S\left(h_{2}\right) \\
= & S(h) \otimes c_{0,(0), 0} \cdot \bar{S}\left(c_{0,(0),-1}\right) g \\
= & S\left(\bar{S}\left(c_{-1}\right)\right) \otimes c_{0,(0), 0} \cdot \bar{S}\left(c_{0,(-1)} c_{0,(0),-1}\right) \\
= & c-1 \otimes c_{0}=\tau(c) .
\end{aligned}
$$

In [12], it is also shown that $r(\gamma) \in U(\mathscr{T}(C))$, and it is straightforward to verify that the $*$-inverse of $r(\gamma)$ is given by

$$
r(\gamma)^{\prime}(c)=S\left(S\left(c_{1}\right)_{1}\right) S\left(c_{0,(1)}\right)_{1} S\left(c_{1}\right)_{2} \otimes c_{0,(0)} \cdot S\left(c_{0,(1)}\right)_{2} S\left(c_{1}\right)_{3} .
$$

Then a routine verification similar to the above one shows that

$$
\ell(r(\gamma))(c)=\gamma(c)
$$

for all $c \in C$. It is easy to show that $\ell(\sigma)=\sigma^{\prime}$ and $r\left(\sigma^{\prime}\right)=\sigma$.

2.2. The crossed coproduct. We recall the following definitions from $[8$, $10]$.

Definition 2.2. Let $C$ be a coalgebra and $H$ a Hopf algebra. It is said that $H$ coacts weakly on $C$ if there is a $k$-linear map $\rho: C \rightarrow H \otimes C ; \rho(c)=c_{[-1]} \otimes \mathcal{C}_{[0]}$ satisfying the following conditions for all $c \in C$ :

$$
\begin{gathered}
c_{[-1]} \otimes c_{[0] 1} \otimes c_{[0] 2}=c_{1[-1]} c_{2[-1]} \otimes c_{1[0]} \otimes c_{2[0]}, \\
\varepsilon_{C}\left(c_{[0]}\right) c_{[-1]}=\varepsilon(c) 1_{H}, \\
\varepsilon_{H}\left(c_{[-1]}\right) c_{[0]}=c .
\end{gathered}
$$

Assume that $H$ coacts weakly on $C$, and let $\alpha: C \rightarrow H \otimes H, \alpha(c)=\alpha_{1}(c) \otimes$ $\alpha_{2}(c)$, be a linear map. Let $C \triangleleft_{\alpha} H$ be the coalgebra whose underlying vector 
space is $C \otimes H$, with comultiplication and counit given by

$$
\begin{gathered}
\Delta_{\alpha}(c \rtimes h)=\left(c_{1} \rtimes c_{2[-1]} \alpha_{1}\left(c_{3}\right) h_{1}\right) \otimes\left(c_{2[0]} \rtimes \alpha_{2}\left(c_{3}\right) h_{2}\right), \\
\varepsilon_{\alpha}(c \rtimes h)=\varepsilon_{C}(c) \varepsilon_{H}(h) .
\end{gathered}
$$

It was pointed out in [10] that $\varepsilon_{\alpha}(c \rtimes h)$ satisfies the counit property if and only if

$$
\left(\varepsilon_{H} \otimes \mathrm{id}\right) \alpha(c)=\left(\operatorname{id} \otimes \varepsilon_{H}\right) \alpha(c)=\varepsilon_{C}(c) 1_{H}
$$

and that $\Delta_{\alpha}$ is coassociative if and only if $\alpha$ satisfies

$$
\begin{gathered}
c_{1[-1]} \alpha_{1}\left(c_{2}\right) \otimes \alpha_{1}\left(c_{1[0]}\right) \alpha_{2}\left(c_{2}\right)_{1} \otimes \alpha_{2}\left(c_{1[0]}\right) \alpha_{2}\left(c_{2}\right)_{2} \\
=\alpha_{1}\left(c_{1}\right) \alpha_{1}\left(c_{2}\right)_{1} \otimes \alpha_{2}\left(c_{1}\right) \alpha_{1}\left(c_{2}\right)_{2} \otimes \alpha_{2}\left(c_{2}\right), \\
c_{1[-1]} \alpha_{1}\left(c_{2}\right) \otimes c_{1[0][-1]} \alpha_{2}\left(c_{2}\right) \otimes c_{1[0][0]} \\
=\alpha_{1}\left(c_{1}\right) c_{2[-1] 1} \otimes \alpha_{2}\left(c_{1}\right) c_{2[-1] 2} \otimes c_{2[0]} .
\end{gathered}
$$

In [10], (2.36) is called the cocycle condition and (2.37) is called the twisted comodule condition. Following [7], we call $\alpha$, satisfying (2.35), (2.36), and (2.37), a Harrison 2-cocycle.

Now, consider two weak $H$-coactions $\rho, \rho^{\prime}: C \rightarrow H \otimes C$, and write

$$
\rho(c)=c_{[-1]} \otimes c_{[0]}, \quad \rho^{\prime}(c)=c_{\langle-1\rangle} \otimes c_{\langle 0\rangle} .
$$

Also consider two 2-cocycles $\alpha, \alpha^{\prime}: C \rightarrow H \otimes H$ corresponding respectively to $\rho$ and $\rho^{\prime}$, and write

$$
\alpha(c)=\alpha_{1}(c) \otimes \alpha_{2}(c), \quad \alpha^{\prime}(c)=\alpha_{1}^{\prime}(c) \otimes \alpha_{2}^{\prime}(c) .
$$

Then we can consider the crossed coproducts $C \rtimes_{\alpha} H$ and $C \rtimes_{\alpha^{\prime}}^{\prime} H$. In the next lemma, we discuss when these are isomorphic.

LEMMA 2.3. Consider a convolution invertible map $u: C \rightarrow H$ satisfying the conditions

$$
\begin{gathered}
c_{\langle-1\rangle} \otimes c_{\langle 0\rangle}=u^{-1}\left(c_{1}\right) c_{2[-1]} u\left(c_{3}\right) \otimes c_{2[0]}, \\
\alpha^{\prime}(c)=u^{-1}\left(c_{1}\right) c_{2[-1]} \alpha_{1}\left(c_{3}\right) u\left(c_{4}\right)_{1} \otimes u^{-1}\left(c_{2[0]}\right) \alpha_{2}\left(c_{3}\right) u\left(c_{4}\right)_{2}
\end{gathered}
$$

for all $c \in C$. Then the map

$$
\phi: C \rtimes_{\alpha^{\prime}}^{\prime} H \longrightarrow C \rtimes_{\alpha} H, \quad \phi\left(c \rtimes^{\prime} h\right)=c_{1} \rtimes u\left(c_{2}\right) h
$$


is a left $C$-colinear right $H$-linear coalgebra isomorphism. Every left $C$-colinear right $H$-linear coalgebra isomorphism between $C \rtimes_{\alpha} H$ and $C \rtimes_{\alpha^{\prime}}^{\prime} H$ is of this type.

Proof. The proof is a dual version of a similar statement for crossed products (see [13]).

It was shown in [12] that the crossed coproduct construction can be viewed as a special case of the twisting construction from Section 2.1. Let $H$ be a Hopf algebra and $C$ a right $H$-module coalgebra, and view $C \otimes H$ as a right $H$-module coalgebra, with the right $H$-action being induced by the multiplication by $H$. It was proved in [12] that there is a bijective correspondence between crossed coproduct structures on $C \otimes H$ and twistings of $C \otimes H$. We recall the description of this bijection.

Consider a weak coaction $\rho$ and a 2-cocycle $\alpha$ giving rise to the crossed coproduct $C \rtimes_{\alpha} H$, and write

$$
\rho(c)=c_{[-1]} \otimes c_{[0]}, \quad \alpha(c)=\alpha_{1}(c) \otimes \alpha_{2}(c) .
$$

The corresponding twisting $\tau: C \otimes H \rightarrow H \otimes C \otimes H$ is defined by

$$
\tau(c \otimes h)=S\left(h_{1}\right) c_{1[-1]} \alpha_{1}\left(c_{2}\right) h_{2} \otimes c_{1[0]} \otimes \alpha_{2}\left(c_{2}\right) h_{3}
$$

Conversely, if $\tau$ is a twisting of $C \otimes H$, then $(C \otimes H)^{\top}=C \triangleleft_{\alpha} H$, with weak coaction $\rho$ and 2-cocycle $\alpha$ given by

$$
\begin{aligned}
& \rho(c)=\left(\mathrm{id} \otimes \mathrm{id} \otimes \varepsilon_{H}\right) \tau(c \otimes 1), \\
& \alpha(c)=\left(\mathrm{id} \otimes \varepsilon_{C} \otimes \mathrm{id}\right) \tau(c \otimes 1) .
\end{aligned}
$$

3. Twisted 2-cocycles. Let $H$ be a Hopf algebra with bijective antipode $S$ and let $\bar{S}$ be the composition inverse of $S$. Take an $H$-module coalgebra $C$, and let $B=C / C H^{+}$.

DEFinition 3.1. A map $\alpha: C \rightarrow H \otimes H, \alpha(c)=\alpha_{1}(c) \otimes \alpha_{2}(c)$ is called a twisted 2-cocycle if the following conditions are satisfied for all $h \in H$ and $c \in C$ :

$$
\begin{aligned}
& \left(\operatorname{id}_{H} \otimes \varepsilon_{H}\right) \alpha(c)=\left(\varepsilon_{H} \otimes \operatorname{id}_{H}\right) \alpha(c)=\varepsilon_{C}(c) 1_{H}, \\
& \alpha(c \cdot h)=S\left(h_{1}\right) \alpha_{1}(c) h_{2} \otimes \bar{S}\left(h_{4}\right) \alpha_{2}(c) h_{3}, \\
& \alpha_{1}\left(c_{1}\right) \alpha_{1}\left(c_{3}\right)_{1} \otimes c_{2} \cdot \alpha_{2}\left(c_{1}\right) \alpha_{1}\left(c_{3}\right)_{2} \otimes \alpha_{2}\left(c_{3}\right) \\
& \quad=\alpha_{1}\left(c_{1}\right) \otimes c_{2} \cdot \alpha_{1}\left(c_{3}\right) \alpha_{2}\left(c_{1}\right)_{1} \otimes \alpha_{2}\left(c_{3}\right) \alpha_{2}\left(c_{1}\right)_{2} .
\end{aligned}
$$

Our first result is the fact that the twisted 2-cocycles can be used to define twistings on $C$. 
Proposition 3.2. With notation as above, if $\alpha: C \rightarrow H \otimes H$ is a twisted 2cocycle, then the map

$$
\tau_{\alpha}: C \longrightarrow H \otimes C, \quad \tau_{\alpha}(c)=\alpha_{1}\left(c_{1}\right) \otimes c_{2} \cdot \alpha_{2}\left(c_{1}\right),
$$

is a twisting of $C$.

Proof. It follows easily from (3.1) that $\tau_{\alpha}$ satisfies the normalizing condition (2.9). Next, we compute

$$
\begin{aligned}
(c \cdot h)_{-1} \otimes(c \cdot h)_{0} & =\alpha_{1}\left((c \cdot h)_{1}\right) \otimes(c \cdot h)_{2} \cdot \alpha_{2}\left(\left(c \cdot h_{2}\right)_{1}\right) \\
& =\alpha_{1}\left(c_{1} \cdot h_{1}\right) \otimes c_{2} \cdot h_{2} \alpha_{2}\left(c_{1} \cdot h_{1}\right) \\
& =S\left(h_{1}\right) \alpha_{1}\left(c_{1}\right) h_{2} \otimes c_{2} \cdot h_{5} \bar{S}\left(h_{4}\right) \alpha_{2}\left(c_{1}\right) h_{3} \\
& =S\left(h_{1}\right) \alpha_{1}\left(c_{1}\right) h_{2} \otimes c_{2} \cdot \alpha_{2}\left(c_{1}\right) h_{3} \\
& =S\left(h_{1}\right) c_{-1} h_{2} \otimes c_{0} \cdot h_{3},
\end{aligned}
$$

and (2.13) follows easily. Finally, we compute the left- and right-hand sides of (2.14):

$$
\begin{aligned}
c_{-1} \otimes & c_{0,1} \cdot c_{0,2,-1} \otimes c_{0,2,0} \\
= & \left(1 \otimes \Delta_{\tau}\right) \tau_{\alpha}(c)=\alpha_{1}\left(c_{1}\right) \otimes\left(c_{2} \cdot \alpha_{2}\left(c_{1}\right)\right)_{1} \cdot \alpha_{1}\left(\left(\left(c_{2} \cdot \alpha_{2}\left(c_{1}\right)\right)_{2}\right)_{1}\right) \\
& \otimes\left(\left(c_{2} \cdot \alpha_{2}\left(c_{1}\right)\right)_{2}\right)_{2} \cdot \alpha_{2}\left(\left(\left(c_{2} \cdot \alpha_{2}\left(c_{1}\right)\right)_{2}\right)_{1}\right) \\
= & \alpha_{1}\left(c_{1}\right) \otimes c_{2} \cdot \alpha_{2}\left(c_{1}\right)_{1} \alpha_{1}\left(c_{3} \cdot \alpha_{2}\left(c_{1}\right)_{2}\right) \\
& \otimes\left(c_{4} \cdot \alpha_{2}\left(c_{1}\right)_{3}\right) \cdot \alpha_{2}\left(c_{3} \cdot \alpha_{2}\left(c_{1}\right)_{2}\right) \\
= & \alpha_{1}\left(c_{1}\right) \otimes c_{2} \cdot \alpha_{2}\left(c_{1}\right)_{1} S\left(\alpha_{2}\left(c_{1}\right)_{2}\right) \alpha_{1}\left(c_{3}\right) \alpha_{2}\left(c_{1}\right)_{3} \\
& \otimes c_{4} \cdot \alpha_{2}\left(c_{1}\right)_{6} \bar{S}\left(\alpha_{2}\left(c_{1}\right)_{5}\right) \alpha_{2}\left(c_{3}\right) \alpha_{2}\left(c_{1}\right)_{4} \\
= & \alpha_{1}\left(c_{1}\right) \otimes c_{2} \cdot \alpha_{1}\left(c_{3}\right) \alpha_{2}\left(c_{1}\right)_{1} \otimes c_{4} \cdot \alpha_{2}\left(c_{3}\right) \alpha_{2}\left(c_{1}\right)_{2}, \\
c_{1,-1} c_{2,-1,1} \otimes c_{1,0} \cdot c_{2,-1,2} \otimes c_{2,0} & \\
= & \sum \alpha_{1}\left(c_{11}\right) \alpha_{1}\left(c_{21}\right)_{1} \otimes c_{12} \cdot \alpha_{2}\left(c_{11}\right) \cdot \alpha_{1}\left(c_{21}\right)_{2} \otimes c_{22} \cdot \alpha_{2}\left(c_{21}\right) \\
= & \alpha_{1}\left(c_{1}\right) \alpha_{1}\left(c_{3}\right)_{1} \otimes c_{2} \cdot \alpha_{2}\left(c_{1}\right) \alpha_{1}\left(c_{3}\right)_{2} \otimes c_{4} \cdot \alpha_{2}\left(c_{3}\right) \\
= & \alpha_{1}\left(c_{1}\right) \otimes c_{2} \cdot \alpha_{1}\left(c_{3}\right) \alpha_{2}\left(c_{1}\right)_{1} \otimes c_{4} \cdot \alpha_{2}\left(c_{3}\right) \alpha_{2}\left(c_{1}\right)_{2} .
\end{aligned}
$$

Thus (2.14) follows, and $\tau_{\alpha}$ is a twisting.

There is also a relation between twisted 2-cocycles and Harrison 2-cocycles. Let $C$ be a right $H$-module coalgebra. Consider the trivial weak coaction $\rho(c)=$ $1 \otimes C$ and $\alpha: C \rightarrow H \otimes H$. Then the cocycle condition (2.36) and the twisted 
comodule condition (2.37) of Definition 2.2 take the following form:

$$
\begin{aligned}
& \alpha_{1}\left(c_{2}\right) \otimes \alpha_{1}\left(c_{1}\right) \alpha_{2}\left(c_{2}\right)_{1} \otimes \alpha_{2}\left(c_{1}\right) \alpha_{2}\left(c_{2}\right)_{2} \\
& =\alpha_{1}\left(c_{1}\right) \alpha_{1}\left(c_{2}\right)_{1} \otimes \alpha_{2}\left(c_{1}\right) \alpha_{1}\left(c_{2}\right)_{2} \otimes \alpha_{2}\left(c_{2}\right), \\
& \alpha_{1}\left(c_{2}\right) \otimes \alpha_{2}\left(c_{2}\right) \otimes c_{1}=\alpha_{1}\left(c_{1}\right) \otimes \alpha_{2}\left(c_{1}\right) \otimes c_{2} .
\end{aligned}
$$

The set of Harrison 2-cocyles corresponding to the trivial weak coaction is denoted by $Z_{\text {Harr }}^{2}(H, C)$. Thus, $Z_{\mathrm{Harr}}^{2}(H, C)$ consists of maps satisfying (2.35), (3.7), and (3.8). The set of twisted 2-cocycles $\alpha^{t}: C \otimes H \rightarrow H \otimes H$ in the sense of Definition 3.1 will be denoted by $Z_{\mathrm{tw}}^{2}(H, C \otimes H)$.

Proposition 3.3. Let $C$ be a right $H$-module coalgebra. There exists a biject in between $Z_{\mathrm{Harr}}^{2}(H, C)$ and $Z_{\mathrm{tw}}^{2}(H, C \otimes H)$.

Proof. Take $\alpha^{t} \in Z_{\mathrm{tw}}^{2}(H, C \otimes H)$ and write

$$
\alpha^{t}(c \otimes h)=\sum \alpha_{1}^{t}(c \otimes h) \otimes \alpha_{2}^{t}(c \otimes h)
$$

For all $c \in C$ and $h \in H$, we have

$$
\begin{aligned}
& \alpha_{1}^{t}\left(c_{1} \otimes h_{1}\right) \alpha_{1}^{t}\left(c_{2} \otimes h_{2}\right)_{1} \otimes \alpha_{2}^{t}\left(c_{1} \otimes h_{1}\right) \alpha_{1}^{t}\left(c_{2} \otimes h_{2}\right)_{2} \otimes \alpha_{2}^{t}\left(c_{2} \otimes h_{2}\right) \\
& \quad=\alpha_{1}^{t}\left(c_{1} \otimes h_{1}\right) \otimes \alpha_{1}^{t}\left(c_{2} \otimes h_{2}\right) \alpha_{2}^{t}\left(c_{1} \otimes h_{1}\right)_{1} \otimes \alpha_{2}^{t}\left(c_{2} \otimes h_{2}\right) \alpha_{2}^{t}\left(c_{1} \otimes h_{1}\right)_{2}
\end{aligned}
$$

Now, define $\alpha: C \rightarrow H \otimes H$ by $\alpha(c)=\alpha^{t}(c \otimes 1)$. It is easy to see that $\alpha$ satisfies (2.35) and (3.8). Using (3.10), we compute

$$
\begin{aligned}
\alpha_{1}\left(c_{2}\right) \otimes \alpha_{1}\left(c_{1}\right) \alpha_{2}\left(c_{2}\right)_{1} \otimes \alpha_{2}\left(c_{1}\right) \alpha_{2}\left(c_{2}\right)_{2} \\
=\alpha_{1}\left(c_{1}\right) \otimes \alpha_{1}\left(c_{2}\right) \alpha_{2}\left(c_{1}\right)_{1} \otimes \alpha_{2}\left(c_{2}\right) \alpha_{2}\left(c_{1}\right)_{2} \\
=\alpha_{1}^{t}\left(c_{1} \otimes 1\right) \otimes \alpha_{1}^{t}\left(c_{2} \otimes 1\right) \alpha_{2}^{t}\left(c_{1} \otimes 1\right)_{1} \otimes \alpha_{2}^{t}\left(c_{2} \otimes 1\right) \alpha_{2}^{t}\left(c_{1} \otimes 1\right)_{2} \\
=\alpha_{1}^{t}\left(c_{1} \otimes 1\right) \alpha_{1}^{t}\left(c_{2} \otimes 1\right)_{1} \otimes \alpha_{2}^{t}\left(c_{1} \otimes 1\right) \alpha_{1}^{t}\left(c_{2} \otimes 1\right)_{2} \otimes \alpha_{2}^{t}\left(c_{2} \otimes 1\right) \\
=\alpha_{1}\left(c_{1}\right) \alpha_{1}\left(c_{2}\right)_{1} \otimes \alpha_{2}\left(c_{1}\right) \alpha_{1}\left(c_{2}\right)_{2} \otimes \alpha_{2}\left(c_{2}\right),
\end{aligned}
$$

and it follows that $\alpha$ also satisfies (3.7).

Conversely, let $\alpha \in Z_{\text {Harr }}^{2}(H, C)$, and define $\alpha^{t}: C \otimes H \rightarrow H \otimes H$ by

$$
\alpha^{t}(c \otimes h)=S\left(h_{1}\right) \alpha_{1}(c) h_{2} \otimes \bar{S}\left(h_{4}\right) \alpha_{2}(c) h_{3} .
$$


We can easily show that $\alpha^{t}$ satisfies conditions (3.1) and (3.2) of Definition 3.1. A straightforward computation shows that (3.3) is also satisfied:

$$
\begin{aligned}
\alpha_{1}^{t}\left(c_{1} \otimes\right. & \left.h_{1}\right) \alpha_{1}^{t}\left(c_{3} \otimes h_{3}\right)_{1} \otimes c_{2} \otimes h_{2} \alpha_{2}^{t}\left(c_{1} \otimes h_{1}\right) \alpha_{1}^{t}\left(c_{3} \otimes h_{3}\right)_{2} \otimes \alpha_{2}^{t}\left(c_{3} \otimes h_{3}\right) \\
= & S h_{1} \alpha_{1}\left(c_{1}\right) h_{2} S\left(h_{7}\right) \alpha_{1}\left(c_{3}\right)_{1} h_{8} \\
& \otimes c_{2} \otimes h_{5} \bar{S}\left(h_{4}\right) \alpha_{2}\left(c_{1}\right) h_{3} S\left(h_{6}\right) \alpha_{1}\left(c_{3}\right)_{2} h_{9} \otimes \bar{S}\left(h_{11}\right) \alpha_{2}\left(c_{3}\right) h_{10} \\
= & S\left(h_{1}\right) \alpha_{1}\left(c_{1}\right) \alpha_{1}\left(c_{3}\right)_{1} h_{2} \otimes c_{2} \otimes \alpha_{2}\left(c_{1}\right) \alpha_{1}\left(c_{3}\right)_{2} h_{3} \otimes \bar{S}\left(h_{5}\right) \alpha_{2}\left(c_{3}\right) h_{4} \\
= & S\left(h_{1}\right) \alpha_{1}\left(c_{3}\right) h_{2} \otimes c_{2} \otimes \alpha_{1}\left(c_{1}\right) \alpha_{2}\left(c_{3}\right)_{1} h_{3} \otimes \bar{S}\left(h_{5}\right) \alpha_{2}\left(c_{1}\right) \alpha_{2}\left(c_{3}\right)_{2} h_{4} \\
= & S\left(h_{1}\right) \alpha_{1}\left(c_{1}\right) h_{2} \otimes c_{2} \otimes \alpha_{1}\left(c_{3}\right) \alpha_{2}\left(c_{1}\right)_{1} h_{3} \otimes \bar{S}\left(h_{5}\right) \alpha_{2}\left(c_{3}\right) \alpha_{2}\left(c_{1}\right)_{2} h_{4} \\
= & S\left(h_{1}\right) \alpha_{1}\left(c_{1}\right) h_{2} \otimes c_{2} \otimes h_{7} S\left(h_{8}\right) \alpha_{1}\left(c_{3}\right) h_{9} \bar{S}\left(h_{6}\right) \alpha_{2}\left(c_{1}\right)_{1} h_{3} \\
& \otimes \bar{S}\left(h_{11}\right) \alpha_{2}\left(c_{3}\right) h_{10} \bar{S}\left(h_{5}\right) \alpha_{2}\left(c_{1}\right)_{2} h_{4} \\
= & \alpha_{1}^{t}\left(c_{1} \otimes h_{1}\right) \otimes c_{2} \otimes h_{2} \alpha_{1}^{t}\left(c_{3} \otimes h_{3}\right) \alpha_{2}^{t}\left(c_{1} \otimes h_{1}\right)_{1} \otimes \alpha_{2}^{t}\left(c_{3} \otimes h_{3}\right) \alpha_{2}^{t}\left(c_{1} \otimes h_{1}\right)_{2} .
\end{aligned}
$$

So it follows that $\alpha^{t}$ is a twisted 2-cocycle. We leave it to the reader to show that the maps between $Z_{\mathrm{Harr}}^{2}(H, C)$ and $Z_{\mathrm{tw}}^{2}(H, C \otimes H)$ defined above are the inverses of each other.

4. Equivalence of twistings. In this section, we will define an equivalence relation on the set of twistings of an $H$-module coalgebra $C$. If a twisting is invertible, then all other twistings in the same equivalence class are also invertible.

Proposition 4.1. Take $\tau, \lambda \in \mathcal{T}(C)$ and use notation (2.24). Consider $v \in$ $\operatorname{Hom}(C, H)$ satisfying the following identities, for all $h \in H, c \in C$ :

$$
\begin{gathered}
\varepsilon_{H} \circ v=\varepsilon_{C}, \quad v(c \cdot h)=S\left(h_{1}\right) v(c) h_{2}, \\
c_{1,(-1)} v\left(c_{2}\right)_{1} \otimes c_{1,(0)} \cdot v\left(c_{2}\right)_{2}=v\left(c_{1}\right) c_{2,-1} \otimes c_{2,0,1} \cdot v\left(c_{2,0,2}\right) .
\end{gathered}
$$

Then $\psi: C^{\top} \rightarrow C^{\lambda}, \psi(c)=c_{1} \cdot v\left(c_{2}\right)$, is a left $B$-colinear and right $H$-linear coalgebra map inducing the identity map on $B$. If $v \in \operatorname{Reg}(C, H)$, then $\psi$ is an isomorphism.

Proof. Using the second identity in (4.1) and $B=C / \mathrm{CH}^{+}$, we can easily prove that $\psi$ is left $B$-colinear and right $H$-linear. Using the first identity in (4.1), we obtain that $\psi$ induces a well-defined map $B \rightarrow B$, which is the identity. In order to prove that $\psi$ is a coalgebra map, we need to check that

$$
\psi\left(c_{1} \cdot c_{2,-1}\right) \otimes \psi\left(c_{2,0}\right)=\psi(c)_{1} \psi(c)_{2,(-1)} \otimes \psi(c)_{2,(0)} .
$$


Again, we compute the left- and right-hand sides and see that they are equal:

$$
\begin{aligned}
\psi(c)_{1} & \psi(c)_{2,(-1)} \otimes \psi(c)_{2,(0)} \\
= & \left(c_{1} \cdot v\left(c_{2}\right)\right)_{1}\left(c_{1} \cdot v\left(c_{2}\right)\right)_{2,(-1)} \otimes\left(c_{1} \cdot v\left(c_{2}\right)\right)_{2,(0)} \\
= & c_{1} \cdot v\left(c_{3}\right)_{1}\left(c_{2} \cdot v\left(c_{3}\right)_{2}\right)_{(-1)} \otimes\left(c_{2} \cdot v\left(c_{3}\right)_{2}\right)_{(0)} \\
= & c_{1} \cdot v\left(c_{3}\right)_{1} S\left(v\left(c_{3}\right)_{2}\right) c_{2,(-1)} v\left(c_{3}\right)_{3} \otimes c_{2,(0)} \cdot v\left(c_{3}\right)_{4} \\
= & c_{1} \cdot c_{2,(-1)} v\left(c_{3}\right)_{1} \otimes c_{2,(0)} \cdot v\left(c_{3}\right)_{2} \\
= & c_{1} \cdot v\left(c_{2}\right) c_{3,-1} \otimes c_{3,0,1} \cdot v\left(c_{3,0,2}\right), \\
\psi\left(c_{1}\right. & \left.\cdot c_{2,-1}\right) \otimes \psi\left(c_{2,0}\right) \\
= & \left(c_{1} \cdot c_{2,-1}\right)_{1} \cdot v\left(\left(c_{1} \cdot c_{2,-1}\right)_{2}\right) \otimes\left(c_{2,0}\right)_{1} \cdot v\left(\left(c_{2,0}\right)_{2}\right) \\
= & c_{1} \cdot\left(c_{3,-1}\right)_{1} v\left(c_{2} \cdot\left(c_{3,-1}\right)_{2}\right) \otimes\left(c_{3,0}\right)_{1} \cdot v\left(\left(c_{3,0}\right)_{2}\right) \\
= & c_{1} \cdot c_{3,-1,1} S\left(c_{3,-1,2}\right) v\left(c_{2}\right) c_{3,-1,3} \otimes c_{3,0,1} \cdot v\left(c_{3,0,2}\right) \\
= & c_{1} \cdot v\left(c_{2}\right) c_{3,-1} \otimes c_{3,0,1} \cdot v\left(c_{3,0,2}\right) .
\end{aligned}
$$

If $v \in \operatorname{Reg}(C, H)$, then its inverse $w$ also satisfies (4.1), and $\varphi: C^{\lambda} \rightarrow C^{\tau}$ defined by

$$
\varphi(c)=c_{1} \cdot w\left(c_{2}\right)
$$

is the inverse of $\psi$.

DefinItion 4.2. It is said that $\tau, \lambda \in \mathscr{T}(C)$ are equivalent if there exists $v \in \operatorname{Reg}(C, H)$ satisfying the conditions of Proposition 4.1. This is denoted by $\tau \sim \lambda$.

LEMMA 4.3. The relation $\sim$ is an equivalence relation on $\mathcal{T}(C)$.

Proof. Clearly, $\tau \sim \tau$ through $v(c)=\varepsilon(c) 1_{H}$.

Next, assume that $\tau \sim \lambda$, and take $v \in \operatorname{Reg}(C, H)$ satisfying (4.1) and (4.2). Equation (4.2) is equivalent to

$$
c_{(-1)} \otimes c_{(0)}=v\left(c_{1}\right) c_{2,-1} v^{-1}\left(c_{3}\right)_{1} \otimes c_{2,0,1} \cdot v\left(c_{2,0,2}\right) v^{-1}\left(c_{3}\right)_{2} .
$$

The inverse $u$ of $v$ satisfies (4.1). It also satisfies (4.2) since

$$
\begin{aligned}
u\left(c_{1}\right) & c_{2,(-1)} \otimes c_{2,(0) 1} \cdot u\left(c_{2,(0), 2}\right) \\
= & u\left(c_{1}\right) v\left(c_{2}\right) c_{3,-1} v^{-1}\left(c_{4}\right)_{1} \otimes c_{3,0,1} \cdot v\left(c_{3,0,3}\right)_{1} v^{-1}\left(c_{4}\right)_{2} \\
& \cdot S\left(v^{-1}\left(c_{4}\right)_{3}\right) S\left(v\left(c_{3,0,3}\right)_{2}\right) u\left(c_{3,0,2}\right) v\left(c_{3,0,3}\right)_{3} v^{-1}\left(c_{4}\right)_{4} \\
= & c_{1,-1} v^{-1}\left(c_{2}\right)_{1} \otimes c_{1,0,1} \cdot u\left(c_{1,0,2}\right) v\left(c_{1,0,3}\right) v^{-1}\left(c_{2}\right)_{2} \\
= & c_{1,-1} u\left(c_{2}\right)_{1} \otimes c_{1,0,1} \cdot u\left(c_{2}\right)_{2},
\end{aligned}
$$

and it follows that $\lambda \sim \tau$. 
Now, assume that $\tau \sim \lambda$ and $\lambda \sim \gamma$, and take the corresponding maps $v, u \in$ $\operatorname{Reg}(C, H)$. Set $w=u * v$, and write

$$
\tau(c)=c_{-1} \otimes c_{0}, \quad \lambda(c)=c_{(-1)} \otimes c_{(0)}, \quad \gamma(c)=c_{[-1]} \otimes c_{[0]} .
$$

It is easily shown that $w$ satisfies (4.1), $v$ satisfies (4.6), and $u$ satisfies

$$
c_{[-1]} \otimes c_{[0]}=u\left(c_{1}\right) c_{2,(-1)} u^{-1}\left(c_{3}\right)_{1} \otimes c_{2,(0), 1} \cdot u\left(c_{2,(0), 2}\right) u^{-1}\left(c_{3}\right)_{2} .
$$

We compute

$$
\begin{aligned}
c_{[-1]} \otimes c_{[0]}=u\left(c_{1}\right) v\left(c_{2}\right) c_{3,-1} v^{-1}\left(c_{4}\right)_{1} u^{-1}\left(c_{5}\right)_{1} \otimes c_{3,0,1} \\
\cdot v\left(c_{3,0,3}\right)_{1} v^{-1}\left(c_{4}\right)_{2} S\left(v^{-1}\left(c_{4}\right)_{3}\right) S\left(v\left(c_{3,0,3}\right)_{2}\right) \\
\cdot u\left(c_{3,0,2}\right) v\left(c_{3,0,3}\right)_{3} v^{-1}\left(c_{4}\right)_{4} u^{-1}\left(c_{5}\right)_{2} \\
=u\left(c_{1}\right) v\left(c_{2}\right) c_{3,-1} v^{-1}\left(c_{4}\right)_{1} u^{-1}\left(c_{5}\right)_{1} \\
\otimes c_{3,0,1} \cdot u\left(c_{3,0,2}\right) v\left(c_{3,0,3}\right) v^{-1}\left(c_{4}\right)_{2} u^{-1}\left(c_{5}\right)_{2} \\
=(u * v)\left(c_{1}\right) c_{2,-1}(u * v)^{-1}\left(c_{3}\right)_{1} \\
\otimes c_{2,0,1} \cdot(u * v)\left(c_{2,0,2}\right)(u * v)^{-1}\left(c_{3}\right)_{2},
\end{aligned}
$$

and this proves that $\tau \sim \gamma$.

THEOREM 4.4. Take $\tau \sim \lambda \in \mathcal{T}(C)$. If $\tau$ is invertible, then $\lambda$ is also invertible.

Proof. Take $v \in \operatorname{Reg}(C, H)$ satisfying the conditions in Proposition 4.1 and let $\psi: C^{\tau} \rightarrow C^{\lambda}$ be the coalgebra isomorphism given by

$$
\psi(c)=c_{1} \cdot v\left(c_{2}\right)
$$

Let $\tau^{-1}$ be the inverse of $\tau$, and write

$$
\tau^{-1}(c)=c_{\langle-1\rangle} \otimes c_{\langle 0\rangle}, \quad \tau(c)=c_{-1} \otimes c_{0}, \quad \lambda(c)=c_{(-1)} \otimes \mathcal{C}_{(0)} .
$$

Define $\mu: C \rightarrow H \otimes C$ by

$$
\begin{aligned}
\mu(c)=c_{[-1]} \otimes c_{[0]}= & \psi^{-1}(c)_{\langle-1\rangle} v^{-1}\left(\psi^{-1}(c)_{\langle 0\rangle 1}\right) v\left(\psi^{-1}(c)_{\langle 0\rangle 3}\right)_{1} \\
& \otimes \psi^{-1}(c)_{\langle 0\rangle} \cdot v\left(\psi^{-1}(c)_{\langle 0\rangle}\right)_{2} .
\end{aligned}
$$

Using the temporary notation $\psi^{-1}(c)_{\langle-1\rangle}=a$ and $\psi^{-1}(c)_{\langle 0\rangle}=b$, it is not hard to prove that $\mu$ is a left inverse of $\lambda$. Indeed, 


$$
\begin{aligned}
(\mu * \lambda)(c) & =(m \otimes \mathrm{id})(\mathrm{id} \otimes \lambda) \mu(c) \\
& =a v^{-1}\left(b_{1}\right) v\left(b_{3}\right)_{1}\left(b_{2} \cdot v\left(b_{3}\right)_{2}\right)_{(-1)} \otimes\left(b_{2} \cdot v\left(b_{3}\right)\right)_{(0)} \\
& =a v^{-1}\left(b_{1}\right) v\left(b_{3}\right)_{1} S\left(v\left(b_{3}\right)_{2}\right) b_{2,(-1)} v\left(b_{3}\right)_{3} \otimes b_{2,(0)} \cdot v\left(b_{3}\right)_{4} \\
& =a v^{-1}\left(b_{1}\right) b_{2,(-1)} v\left(b_{3}\right)_{1} \otimes b_{2,(0)} \cdot v\left(b_{3}\right)_{2} \\
& =a v^{-1}\left(b_{1}\right) v\left(b_{2}\right) b_{3,-1} \otimes b_{3,0,1} \cdot v\left(b_{3,0,2}\right) \\
& =a b_{-1} \otimes b_{0,1} \cdot v\left(b_{0,2}\right)=1 \otimes \psi^{-1}(c)_{1} \cdot v\left(\psi^{-1}(c)_{2}\right) \\
& =1 \otimes \psi\left(\psi^{-1}(c)\right)=1 \otimes c=\sigma(c) .
\end{aligned}
$$

The proof of the fact that $\mu$ is also a right inverse of $\lambda$ is much more technical. From the fact that $v$ is invertible, and using (4.2), we obtain

$$
\lambda(c)=c_{(-1)} \otimes c_{(0)}=v\left(c_{1}\right) c_{2,-1} v^{-1}\left(c_{3}\right)_{1} \otimes c_{2,0,1} \cdot v\left(c_{2,0,2}\right) v^{-1}\left(c_{3}\right)_{2} .
$$

Now, set $\psi^{-1}(c)=c_{1} \cdot v^{-1}\left(c_{2}\right)$. We compute

$$
\begin{aligned}
& (\lambda * \mu)(c)=(m \otimes \mathrm{id})(\mathrm{id} \otimes \mu) \lambda(c) \\
& =v\left(c_{1}\right) c_{2,-1} v^{-1}\left(c_{3}\right)_{1}\left(c_{2,0,1} \cdot v\left(c_{2,0,2}\right) v^{-1}\left(c_{3}\right)_{2}\right)_{[-1]} \\
& \otimes\left(c_{2,0,1} \cdot v\left(c_{2,0,2}\right) v^{-1}\left(c_{3}\right)_{2}\right)_{[0]} \\
& =v\left(c_{1}\right) c_{2,-1} v^{-1}\left(c_{3}\right)_{1} S\left(v^{-1}\left(c_{3}\right)_{2}\right) S\left(v\left(c_{2,0,2}\right)_{1}\right)\left(c_{2,0,1}\right)_{[-1]} \\
& \cdot v\left(c_{2,0,2}\right)_{2} v^{-1}\left(c_{3}\right)_{3} \otimes\left(c_{2,0,1}\right)_{[0]} \cdot v\left(c_{2,0,2}\right)_{3} v^{-1}\left(c_{3}\right)_{4} \\
& =v\left(c_{1}\right) c_{2,-1} S\left(v\left(c_{2,0,2}\right)_{1}\right)\left(c_{2,0,1}\right)_{[-1]} v\left(c_{2,0,2}\right)_{2} v^{-1}\left(c_{3}\right)_{1} \\
& \otimes\left(c_{2,0,1}\right)_{[0]} \cdot v\left(c_{2,0,2}\right)_{3} v^{-1}\left(c_{3}\right)_{2} \\
& =v\left(c_{1}\right) c_{2,-1} S\left(v\left(c_{2,0,2}\right)_{1}\right) \psi^{-1}\left(c_{2,0,1}\right)_{\langle-1\rangle} v^{-1}\left(\psi^{-1}\left(c_{2,0,1}\right)_{\langle 0\rangle .1}\right) \\
& \cdot v\left(\psi^{-1}\left(c_{2,0,1}\right)_{\langle 0\rangle, 3}\right)_{1} v\left(c_{2,0,2}\right)_{2} v^{-1}\left(c_{3}\right)_{1} \otimes \psi^{-1}\left(c_{2,0,1}\right)_{\langle 0\rangle, 2} \\
& \cdot v\left(\psi^{-1}\left(c_{2,0,1}\right)_{\langle 0\rangle, 3}\right)_{2} v\left(c_{2,0,2}\right)_{3} v^{-1}\left(c_{3}\right)_{2} \\
& =v\left(c_{1}\right) c_{2,-1} S\left(v\left(c_{2,0,3}\right)_{1}\right)\left(c_{2,0,1} \cdot v^{-1}\left(c_{2,0,2}\right)\right)_{\langle-1\rangle} \\
& \cdot v^{-1}\left(\left(c_{2,0,1} \cdot v^{-1}\left(c_{2,0,2}\right)\right)_{\langle 0\rangle 1}\right) v\left(\left(c_{2,0,1} \cdot v^{-1}\left(c_{2,0,2}\right)\right)_{\langle 0\rangle 3}\right)_{1} \\
& \cdot v\left(c_{2,0,3}\right)_{2} v^{-1}\left(c_{3}\right)_{1} \otimes\left(c_{2,0,1} \cdot v^{-1}\left(c_{2,0,2}\right)\right)_{\langle 0\rangle} \\
& \cdot v\left(\left(c_{2,0,1} \cdot v^{-1}\left(c_{2,0,2}\right)\right)_{\langle 0\rangle 3}\right)_{2} v\left(c_{2,0,3}\right)_{3} v^{-1}\left(c_{3}\right)_{2} \\
& =v\left(c_{1}\right) c_{2,-1} S\left(v\left(c_{2,0,3}\right)_{1}\right) S\left(v^{-1}\left(c_{2,0,2}\right)_{1}\right) c_{2,0,1,\langle-1\rangle} v^{-1}\left(c_{2,0,2}\right)_{2} \\
& \cdot v^{-1}\left(\left(c_{2,0,1,\langle 0\rangle} \cdot v^{-1}\left(c_{2,0,2}\right)_{3}\right)_{1}\right) v\left(\left(c_{2,0,1,\langle 0\rangle} \cdot v^{-1}\left(c_{2,0,2}\right)_{3}\right)_{3}\right)_{1} \\
& \cdot v\left(c_{2,0,3}\right)_{2} v^{-1}\left(c_{3}\right)_{1} \otimes\left(c_{2,0,1,\langle 0\rangle} \cdot v^{-1}\left(c_{2,0,2}\right)_{3}\right)_{2} \\
& \cdot v\left(\left(c_{2,0,1,\langle 0\rangle} \cdot v^{-1}\left(c_{2,0,2}\right)_{3}\right)_{3}\right)_{2} v\left(c_{2,0,3}\right)_{3} v^{-1}\left(c_{3}\right)_{2}
\end{aligned}
$$




$$
\begin{aligned}
& =v\left(c_{1}\right) c_{2,-1} S\left(v^{-1}\left(c_{2,0,2}\right)_{1} v\left(c_{2,0,3}\right)_{1}\right) c_{2,0,1,\langle-1\rangle} v^{-1}\left(c_{2,0,2}\right)_{2} \\
& \cdot v^{-1}\left(c_{2,0,1,\langle 0\rangle, 1} \cdot v^{-1}\left(c_{2,0,2}\right)_{3}\right) v\left(c_{2,0,1,\langle 0\rangle, 3} \cdot v^{-1}\left(c_{2,0,2}\right)_{5}\right)_{1} v\left(c_{2,0,3}\right)_{2} \\
& \cdot v^{-1}\left(c_{3}\right)_{1} \otimes c_{2,0,1,\langle 0\rangle, 2} \cdot v^{-1}\left(c_{2,0,2}\right)_{4} \\
& \cdot v\left(c_{2,0,1,\langle 0\rangle, 3} \cdot v^{-1}\left(c_{2,0,2}\right)_{5}\right)_{2} v\left(c_{2,0,3}\right)_{3} v^{-1}\left(c_{3}\right)_{2} \\
& =v\left(c_{1}\right) c_{2,-1} S\left(v^{-1}\left(c_{2,0,2}\right)_{1} v\left(c_{2,0,3}\right)_{1}\right) c_{2,0,1,\langle-1\rangle} v^{-1}\left(c_{2,0,2}\right)_{2} \\
& \cdot S\left(v^{-1}\left(c_{2,0,2}\right)_{3}\right) v^{-1}\left(c_{2,0,1,\langle 0\rangle, 1}\right) v^{-1}\left(c_{2,0,2}\right)_{4} S\left(v^{-1}\left(c_{2,0,2}\right)_{6}\right)_{1} \\
& \cdot v\left(c_{2,0,1,\langle 0\rangle, 3}\right)_{1}\left(v^{-1}\left(c_{2,0,2}\right)_{7}\right)_{1} v\left(c_{2,0,3}\right)_{2} v^{-1}\left(c_{3}\right)_{1} \\
& \otimes c_{2,0,1,\langle 0\rangle, 2} \cdot v^{-1}\left(c_{2,0,2}\right)_{5} S\left(v^{-1}\left(c_{2,0,2}\right)_{6}\right)_{2} \\
& \cdot v\left(c_{2,0,1,\langle 0\rangle, 3}\right)_{2}\left(v^{-1}\left(c_{2,0,2}\right)_{7}\right)_{2} v\left(c_{2,0,3}\right)_{3} v^{-1}\left(c_{3}\right)_{2} \\
& =v\left(c_{1}\right) c_{2,-1} S\left(v^{-1}\left(c_{2,0,2}\right){ }_{1} v\left(c_{2,0,3}\right)_{1}\right) c_{2,0,1,\langle-1\rangle} v^{-1}\left(c_{2,0,2}\right)_{2} S\left(v^{-1}\left(c_{2,0,2}\right)_{3}\right) \\
& \cdot v^{-1}\left(c_{2,0,1,\langle 0\rangle, 1}\right) v^{-1}\left(c_{2,0,2}\right)_{4} S\left(v^{-1}\left(c_{2,0,2}\right)_{7}\right) v\left(c_{2,0,1,\langle 0\rangle, 3}\right)_{1} \\
& \cdot v^{-1}\left(c_{2,0,2}\right)_{8} v\left(c_{2,0,3}\right)_{2} v^{-1}\left(c_{3}\right)_{1} \otimes c_{2,0,1,\langle 0\rangle, 2} \cdot v^{-1}\left(c_{2,0,2}\right)_{5} \\
& \cdot S\left(v^{-1}\left(c_{2,0,2}\right)_{6}\right) v\left(c_{2,0,1,\langle 0\rangle, 3}\right)_{2} v^{-1}\left(c_{2,0,2}\right)_{9} v\left(c_{2,0,3}\right)_{3} v^{-1}\left(c_{3}\right)_{2} \\
& =v\left(c_{1}\right) c_{2,-1} S\left(v^{-1}\left(c_{2,0,2}\right)_{1} v\left(c_{2,0,3}\right)_{1}\right) c_{2,0,1,\langle-1\rangle} v^{-1}\left(c_{2,0,1,\langle 0\rangle, 1}\right) \\
& \cdot v\left(c_{2,0,1,\langle 0\rangle, 3}\right)_{1} v^{-1}\left(c_{2,0,2}\right)_{2} v\left(c_{2,0,3}\right)_{2} v^{-1}\left(c_{3}\right)_{1} \\
& \otimes c_{2,0,1,\langle 0\rangle, 2} \cdot v\left(c_{2,0,1,\langle 0\rangle, 3}\right)_{2} v^{-1}\left(c_{2,0,2}\right)_{3} v\left(c_{2,0,3}\right)_{3} v^{-1}\left(c_{3}\right)_{2} \\
& =v\left(c_{1}\right) c_{2,-1} c_{2,0,\langle-1\rangle} v^{-1}\left(c_{2,0,\langle 0\rangle, 1}\right) v\left(c_{2,0,\langle 0\rangle, 3}\right)_{1} v^{-1}\left(c_{3}\right)_{1} \\
& \otimes c_{2,0,\langle 0\rangle, 2} \cdot v\left(c_{2,0,\langle 0\rangle, 3}\right)_{2} v^{-1}\left(c_{3}\right)_{2} \\
& =v\left(c_{1}\right) v^{-1}\left(c_{2,1}\right) v\left(c_{2,3}\right)_{1} v^{-1}\left(c_{3}\right)_{1} \otimes c_{2,2} \cdot v\left(c_{2,3}\right)_{2} v^{-1}\left(c_{3}\right)_{2} \\
& =v\left(c_{1}\right) v^{-1}\left(c_{2}\right) v\left(c_{4}\right)_{1} v^{-1}\left(c_{5}\right)_{1} \otimes c_{3} \cdot v\left(c_{4}\right)_{2} v^{-1}\left(c_{5}\right)_{2} \\
& =1 \otimes c=\sigma(c) \text {, }
\end{aligned}
$$

and it follows that $\lambda$ is convolution invertible.

THEOREM 4.5. Let $C$ be a right $H$-comodule algebra and consider $\tau, \lambda \in$ $\mathcal{T}(C \otimes H)$. Then $\tau$ and $\lambda$ are equivalent in the sense of Definition 4.2 if and only if there is a left $C$-colinear and right $H$-linear coalgebra isomorphism between the crossed coproducts $C \triangleleft_{\alpha} H, \rho$ and $C \rtimes_{\alpha^{\prime}}^{\prime} H, \rho^{\prime}$ corresponding to $\tau$ and $\lambda$.

Proof. Consider (2.38). If $\tau \sim \lambda$, then there exists $v \in \operatorname{Reg}(C \otimes H, H)$ satisfying (4.1) and (4.2). Define

$$
u: C \longrightarrow H, \quad u(c)=v^{-1}(c \otimes 1) .
$$


If we can show that $u$ satisfies (2.40) and (2.41), then one implication is proved by Lemma 2.3. It follows from (4.2) that

$$
\begin{aligned}
& \left(c_{1} \otimes 1\right)_{(-1)} v\left(c_{2} \otimes 1\right)_{1} \otimes\left(c_{1} \otimes 1\right)_{(0)} \cdot v\left(c_{2} \otimes 1\right)_{2} \\
& \quad=v\left(c_{1} \otimes 1\right)\left(c_{2} \otimes 1\right)_{-1} \otimes\left(c_{2} \otimes 1\right)_{0,1} \cdot v\left(\left(c_{2} \otimes 1\right)_{0,2}\right) .
\end{aligned}
$$

Applying $1 \otimes 1 \otimes \varepsilon$ to both sides, we find

$$
\begin{aligned}
& \left(c_{1} \otimes 1\right)_{(-1)} v\left(c_{2} \otimes 1\right) \otimes(1 \otimes \varepsilon)\left(c_{1} \otimes 1\right)_{(0)} \\
& \quad=v\left(c_{1} \otimes 1\right)\left(c_{2} \otimes 1\right)_{-1} \otimes(1 \otimes \varepsilon)\left(c_{2} \otimes 1\right)_{0},
\end{aligned}
$$

and using (2.45), we obtain

$$
c_{\langle-1\rangle} \otimes c_{\langle 0\rangle}=u^{-1}\left(c_{1}\right) c_{2[-1]} u\left(c_{3}\right) \otimes c_{2[0]} .
$$

So $u$ satisfies (2.40).

Applying $1 \otimes \varepsilon \otimes 1$ to both sides of (4.18), we find

$$
\begin{aligned}
\alpha^{\prime}(c)= & v\left(c_{1} \otimes 1\right)\left(c_{2} \otimes 1\right)_{-1} v^{-1}\left(c_{3} \otimes 1\right)_{1} \\
& \otimes(\varepsilon \otimes 1)\left(\left(c_{2} \otimes 1\right)_{0,1} \cdot v\left(\left(c_{2} \otimes 1\right)_{0,2}\right) v^{-1}\left(c_{3} \otimes 1\right)_{2}\right) .
\end{aligned}
$$

It follows from (2.44) that

$$
\begin{aligned}
& (c \otimes 1)_{0}=c_{1[0]} \otimes \alpha_{2}\left(c_{2}\right), \\
& (\varepsilon \otimes 1)\left(\left(c_{2} \otimes 1\right)_{0,1} \cdot v\left(\left(c_{2} \otimes 1\right)_{0,2}\right) v^{-1}\left(c_{3} \otimes 1\right)_{2}\right) \\
& \quad=(\varepsilon \otimes 1)\left(c_{2,[0], 1} \otimes \alpha_{2}\left(c_{3}\right)_{1} v\left(c_{2,[0], 2} \otimes \alpha_{2}\left(c_{3}\right)_{2}\right) v^{-1}\left(c_{4} \otimes 1\right)_{2}\right) \\
& \quad=\alpha_{2}\left(c_{3}\right)_{1} v\left(c_{2,[0]} \otimes \alpha_{2}\left(c_{3}\right)_{2}\right) v^{-1}\left(c_{4} \otimes 1\right)_{2} \\
& =v\left(c_{2,[0]} \otimes 1\right) \alpha_{2}\left(c_{3}\right) v^{-1}\left(c_{4} \otimes 1\right)_{2} \\
& =v\left((1 \otimes \varepsilon)\left(c_{2} \otimes 1\right)_{0} \otimes 1\right)(\varepsilon \otimes 1)\left(c_{3} \otimes 1\right)_{0} v^{-1}\left(c_{4} \otimes 1\right)_{2} .
\end{aligned}
$$

So,

$$
\begin{aligned}
\alpha^{\prime}(c)= & v\left(c_{1} \otimes 1\right)\left(c_{2} \otimes 1\right)_{-1}\left(c_{3} \otimes 1\right)_{-1} v^{-1}\left(c_{4} \otimes 1\right)_{1} \\
& \otimes v\left((1 \otimes \varepsilon)\left(c_{2} \otimes 1\right)_{0} \otimes 1\right)(\varepsilon \otimes 1)\left(c_{3} \otimes 1\right)_{0} v^{-1}\left(c_{4} \otimes 1\right)_{2} \\
= & u^{-1}\left(c_{1}\right) c_{2[-1]} \alpha_{1}\left(c_{3}\right) u\left(c_{4}\right)_{1} \otimes u^{-1}\left(c_{2[0]}\right) \alpha_{2}\left(c_{3}\right) u\left(c_{4}\right)_{2},
\end{aligned}
$$

and (2.41) follows. 
Conversely, assume that the two crossed coproducts are isomorphic. By Lemma 2.3, there exists $u \in \operatorname{Reg}(C, H)$ satisfying (2.40) and (2.41). Define

$$
v: C \otimes H \longrightarrow H, \quad v(c \otimes h)=S\left(h_{1}\right) u^{-1}(c) h_{2} .
$$

Then

$$
\begin{aligned}
\varepsilon_{H} v(c \otimes h) & =\varepsilon\left(S\left(h_{1}\right) u^{-1}(c) h_{2}\right)=\varepsilon(c) \varepsilon(h), \\
v((c \otimes h) \cdot g) & =v(c \otimes h g)=S(h g)_{1} u^{-1}(c)(h g)_{2} \\
& =S\left(g_{1}\right) S\left(h_{1}\right) u^{-1}(c) h_{2} g_{2}=S\left(g_{1}\right) v(c \otimes h) g_{2} .
\end{aligned}
$$

So,

$$
\begin{aligned}
\lambda(c \otimes h)= & (c \otimes h)_{1} \otimes(c \otimes h)_{0} \\
= & S\left(h_{1}\right) c_{1,\langle-1\rangle} \alpha_{1}^{\prime}\left(c_{2}\right) h_{2} \otimes c_{1,\langle 0\rangle} \otimes \alpha_{2}^{\prime}\left(c_{2}\right) h_{3} \\
= & S\left(h_{1}\right) u^{-1}\left(c_{1}\right) c_{2,[-1]} u\left(c_{3}\right) u^{-1}\left(c_{4}\right) c_{5,[-1]} \alpha_{1}\left(c_{6}\right) u\left(c_{7}\right)_{1} h_{2} \\
& \otimes c_{2,[0]} \otimes u^{-1}\left(c_{5,[0]}\right) \alpha_{2}\left(c_{6}\right) u\left(c_{7}\right)_{2} h_{3} \\
= & S\left(h_{1}\right) u^{-1}\left(c_{1}\right) c_{2,[-1]} \alpha_{1}\left(c_{3}\right) u\left(c_{4}\right)_{1} h_{2} \otimes c_{2,[0], 1} \\
& \otimes u^{-1}\left(c_{2,[0], 2}\right) \alpha_{2}\left(c_{3}\right) u\left(c_{4}\right)_{2} h_{3} \\
= & S\left(h_{1}\right) u^{-1}\left(c_{1}\right) h_{2} S\left(h_{3}\right) c_{2,[-1]} \alpha_{1}\left(c_{3}\right) h_{4} S\left(h_{7}\right) u\left(c_{4}\right)_{1} h_{8} \\
& \otimes c_{2,[0], 1} \otimes u^{-1}\left(c_{2,[0], 2}\right) \alpha_{2}\left(c_{3}\right) h_{5} S\left(h_{6}\right) u\left(c_{4}\right)_{2} h_{9} \\
= & S\left(h_{1}\right) u^{-1}\left(c_{1}\right) h_{2} S\left(h_{3}\right) c_{2,[-1]} \alpha_{1}\left(c_{3}\right) h_{4} S\left(h_{9}\right) u\left(c_{4}\right)_{1} h_{10} \otimes c_{2,[0], 1} \\
& \otimes \alpha_{2}\left(c_{3}\right)_{1} h_{5} S\left(h_{6}\right) S\left(\alpha_{2}\left(c_{3}\right)_{2}\right) u^{-1}\left(c_{2,[0], 2}\right) \alpha_{2}\left(c_{3}\right)_{3} h_{7} S\left(h_{8}\right) u\left(c_{4}\right)_{2} h_{11} \\
= & v\left(c_{1} \otimes h_{1}\right)\left(c_{2} \otimes h_{2}\right)_{-1} v^{-1}\left(c_{3} \otimes h_{3}\right)_{1} \\
& \otimes\left(c_{2} \otimes h_{2}\right)_{0,1} \cdot v\left(\left(c_{2} \otimes h_{2}\right)_{0,2}\right) v^{-1}\left(c_{3} \otimes h_{3}\right)_{2} .
\end{aligned}
$$

This shows that $\tau \sim \lambda$.

5. Twisting Hopf-Galois coextensions. Let $H$ be a Hopf algebra with bijective antipode $S$, and $C$ a right $H$-module coalgebra. As before, we use the following notation:

$$
B=C / I, \quad I=\{c(h-\varepsilon(h)) \mid h \in H, c \in C\} .
$$

For $\tau \in \mathscr{T}(C)$, we have that $C^{\top} / I^{\tau}=C / I=B$.

Now, assume that $C / B$ is an $H$-Galois coextension (see [5]). This means that the canonical map

$$
\beta: C \otimes H \longrightarrow C \square_{B} C, \quad \beta(c \otimes h)=c_{1} \otimes c_{2} \cdot h,
$$


is a bijection. Recall that, in this situation, $C \square_{B} C$ is the cotensor product

$$
C \square_{B} C=\left\{\Sigma_{i} C_{i} \otimes d_{i} \in C \otimes C \mid \Sigma_{i} c_{i_{1}} \otimes \pi\left(c_{i_{2}}\right) \otimes d_{i}=\Sigma_{i} C_{i} \otimes \pi\left(d_{i_{1}}\right) \otimes d_{i_{2}}\right\},
$$

where $\pi: C \rightarrow B$ is the natural epimorphism.

LEMMA 5.1. With notation as above, consider the map

$$
\beta^{\prime}: C \otimes H \rightarrow C \square_{B} C, \quad \beta^{\prime}(c \otimes h)=c_{1} \cdot h \otimes c_{2} .
$$

If the antipode $S$ is bijective, then $\beta$ is bijective (resp., injective, resp., surjective) if and only if $\beta^{\prime}$ is bijective (resp., injective, resp., surjective).

Proof. The map

$$
\phi: C \otimes H \longrightarrow C \otimes H, \quad \phi(c \otimes h)=c \cdot h_{1} \otimes S\left(h_{2}\right)
$$

is a bijection with inverse

$$
\phi^{-1}(c \otimes h)=c \cdot h_{2} \otimes \bar{S} h_{1}
$$

Then the statement follows from the fact that $\beta^{\prime}=\beta \circ \phi$.

THEOREM 5.2. Take $\tau \in U(\mathscr{T}(C))$. Then $C^{\tau} / B$ is an $H$-Galois coextension if and only if $C / B$ is an $H$-Galois coextension.

Proof. Let $\lambda$ be the inverse of $\tau$. As before, we use the notation (2.24). Let $\beta^{\tau}$ be the canonical map corresponding to the coextension $C^{\tau} / B$, that is,

$$
\beta^{\tau}(c \otimes h)=c_{1} \cdot c_{2,-1} h \otimes c_{2,0} .
$$

Consider the following diagram:

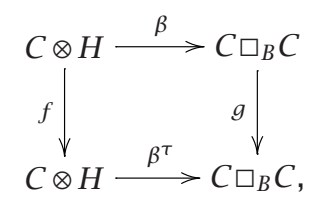

where

$$
f(c \otimes h)=c_{0} \otimes \bar{S}\left(c_{-1}\right) h, \quad g(c \otimes d)=c_{0} \cdot \bar{S}\left(c_{-1}\right) \otimes d,
$$

$f$ and $g$ are bijections, with inverses given by

$$
f^{-1}(c \otimes h)=c_{(0)} \otimes \bar{S}\left(c_{(-1)}\right) h, \quad g^{-1}(c \otimes d)=c_{(0)} \cdot \bar{S}\left(c_{(-1)}\right) \otimes d .
$$


We can also compute

$$
\begin{aligned}
\beta^{\tau} f(c \otimes h) & =\beta^{\top}\left(c_{0} \otimes \bar{S}\left(c_{-1}\right) h\right) \\
& =c_{0,1} \cdot c_{0,2,-1} \bar{S}\left(c_{-1}\right) h \otimes c_{0,2,0} \\
& =c_{1,0} \cdot c_{2,-1,2} \bar{S}\left(c_{1,-1} c_{2,-1,1}\right) h \otimes c_{2,0} \\
& =c_{1,0} \cdot c_{2,-1,2} \bar{S}\left(c_{2,-1,1}\right) \bar{S}\left(c_{1,-1}\right) h \otimes c_{2,0} \\
& =c_{1,0} \cdot \bar{S}\left(c_{1,-1}\right) h \otimes c_{2} \\
& =c_{1,0} \cdot h_{3} \bar{S}\left(h_{2}\right) \bar{S}\left(c_{1,-1}\right) h_{1} \otimes c_{2} \\
& =c_{1,0} \cdot h_{3} \bar{S}\left(S\left(h_{1}\right) c_{1,-1} h_{2}\right) \otimes c_{2} \\
& =\left(c_{1} \cdot h\right)_{0} \cdot \bar{S}\left(\left(c_{1} \cdot h\right)_{-1}\right) \otimes c_{2} \\
& =g\left(c_{1} \cdot h \otimes c_{2}\right)=g \beta(c \otimes h) .
\end{aligned}
$$

This shows that (5.8) is commutative and it follows that $\beta$ is bijective if and only if $\beta^{\top}$ is bijective.

THEOREM 5.3. Let $C / B$ be an $H$-Galois coextension and take $\tau, \lambda \in \mathscr{T}(C)$. Every left B-colinear and right $H$-linear coalgebra map

$$
\psi: C^{\tau} \rightarrow C^{\lambda}
$$

is of the form $\psi(c)=c_{1} \cdot v\left(c_{2}\right)$, where $v \in \operatorname{Hom}(C, H)$ satisfies conditions (4.1) and (4.2) of Proposition 4.1. If $\psi$ is an isomorphism, then $v \in \operatorname{Reg}(C, H)$.

Proof. We use the notation (2.24). As in [5], we consider the map

$$
\bar{\tau}=(\varepsilon \otimes 1) \beta^{-1}: C \square_{B} C \longrightarrow H .
$$

We introduce the notation $c \diamond d=\bar{\tau}(c \otimes d)$. Then we have the following properties:

$$
\begin{aligned}
\varepsilon_{H}(c \diamond d) & =\varepsilon_{C}(c) \varepsilon_{C}(d), \\
(c \diamond d) h & =c \diamond(d \cdot h), \\
(c \cdot h) \diamond d & =S(h)(c \diamond d), \\
c_{1} \cdot\left(c_{2} \diamond d\right) & =\varepsilon(c) d .
\end{aligned}
$$

The map

$$
v: C \rightarrow H, \quad v(c)=c_{1} \diamond \psi\left(c_{2}\right)
$$

satisfies the property

$$
c_{1} \cdot v\left(c_{2}\right)=c_{1} \cdot\left(c_{2} \diamond \psi\left(c_{3}\right)\right)=\psi(c) .
$$


Since $\psi$ is a coalgebra,

$$
\varepsilon_{H} v(c)=\varepsilon_{H}\left(c_{1} \diamond \psi\left(c_{2}\right)\right)=\varepsilon(\psi(c))=\varepsilon(c),
$$

and it follows that $\varepsilon_{H} \circ v=\varepsilon_{C}$.

It follows from (5.16) and (5.17) that

$$
\begin{aligned}
v(c \cdot h) & =(c \cdot h)_{1} \diamond \psi\left((c \cdot h)_{2}\right)=c_{1} \cdot h_{1} \diamond \psi\left(c_{2} \cdot h_{2}\right) \\
& =S\left(h_{1}\right)\left(c_{1} \diamond \psi\left(c_{2}\right)\right) h_{2}=S\left(h_{1}\right) v(c) h_{2} .
\end{aligned}
$$

Since $\psi$ is a coalgebra map, we have that

$$
\begin{aligned}
& \psi\left(c_{1} \cdot c_{2,-1}\right) \otimes \psi\left(c_{2,0}\right)=\psi(c)_{1} \psi(c)_{2,(-1)} \otimes \psi(c)_{2,(0)}, \\
& c_{1} \cdot v\left(c_{2}\right) c_{3,-1} \otimes c_{3,0,1} \cdot v\left(c_{3,0,2}\right) \\
& \quad=\left(c_{1} \cdot v\left(c_{2}\right)\right)_{1}\left(c_{1} \cdot v\left(c_{2}\right)\right)_{2,(-1)} \otimes\left(c_{1} \cdot v\left(c_{2}\right)\right)_{2,(0)} \\
& \quad=c_{1} \cdot v\left(c_{3}\right)_{1}\left(c_{2} \cdot v\left(c_{3}\right)_{2}\right)_{(-1)} \otimes\left(c_{2} \cdot v\left(c_{3}\right)_{2}\right)_{(0)} \\
& =c_{1} \cdot c_{2,(-1)} v\left(c_{3}\right)_{1} \otimes c_{2,(0)} \cdot v\left(c_{3}\right)_{2},
\end{aligned}
$$

which is equivalent to

$$
\begin{aligned}
c_{1} \otimes c_{2} & \cdot v\left(c_{3}\right) c_{4,-1} \otimes c_{4,0,1} \cdot v\left(c_{4,0,2}\right) \\
& =c_{1} \otimes c_{2} \cdot c_{3,(-1)} v\left(c_{4}\right)_{1} \otimes c_{3,(0)} \cdot v\left(c_{4}\right)_{2} .
\end{aligned}
$$

After we apply $\beta^{-1}$ to both sides, we obtain

$$
\begin{gathered}
c_{1} \otimes v\left(c_{2}\right) c_{3,-1} \otimes c_{3,0,1} \cdot v\left(c_{3,0,2}\right)=c_{1} \otimes c_{2,(-1)} v\left(c_{3}\right)_{1} \otimes c_{2,(0)} \cdot v\left(c_{3}\right)_{2}, \\
v\left(c_{1}\right) c_{2,-1} \otimes c_{2,0,1} \cdot v\left(c_{2,0,2}\right)=c_{1,(-1)} v\left(c_{2}\right)_{1} \otimes c_{1,(0)} \cdot v\left(c_{2}\right)_{2} .
\end{gathered}
$$

If $\psi$ is an isomorphism, then $\psi^{-1}: C^{\lambda} \rightarrow C^{\tau}$ is a left $B$-colinear and right $H$ linear coalgebra map. Then we have a map $w: C \rightarrow H$ satisfying (4.1) and (4.2) such that

$$
\psi^{-1}(c)=c_{1} \cdot w\left(c_{2}\right)
$$

For all $c \in C$, we have that

$$
c=c_{1} \cdot v\left(c_{2}\right) w\left(c_{3}\right)=c_{1} \cdot w\left(c_{2}\right) v\left(c_{3}\right)
$$

Proceeding as in the proof of (5.24), we find that $v$ is convolution invertible.

ACKNOWLEDGment. This research was supported by the Project G.0278.01 "Construction and Applications of Noncommutative Geometry: From Algebra to Physics" of FWO Vlaanderen, and by the bilateral Project BIL99/43 "New Computational, Geometric, and Algebraic Methods Applied to Quantum Groups and Differential Operators" of the Flemish and Chinese governments. 


\section{REFERENCES}

[1] M. Beattie, C.-Y. Chen, and J. J. Zhang, Twisted Hopf comodule algebras, Comm. Algebra 24 (1996), no. 5, 1759-1775.

[2] M. Beattie and B. Torrecillas, Twistings and Hopf Galois extensions, J. Algebra 232 (2000), no. 2, 673-696.

[3] R. J. Blattner, M. Cohen, and S. Montgomery, Crossed products and inner actions of Hopf algebras, Trans. Amer. Math. Soc. 298 (1986), no. 2, 671-711.

[4] R. J. Blattner and S. Montgomery, Crossed products and Galois extensions of Hopf algebras, Pacific J. Math. 137 (1989), no. 1, 37-54.

[5] T. Brzeziński and P. M. Hajac, Coalgebra extensions and algebra coextensions of Galois type, Comm. Algebra 27 (1999), no. 3, 1347-1367.

[6] S. Caenepeel, Harrison cocycles and the group of Galois coobjects, Algèbre Non Commutative, Groupes Quantiques et Invariants (Reims, 1995), Sémin. Congr., vol. 2, Société Mathématique de France, Paris, 1997, pp. 83-101.

[7] S. Caenepeel, S. Dăscălescu, G. Militaru, and F. Panaite, Coalgebra deformations of bialgebras by Harrison cocycles, copairings of Hopf algebras and double crosscoproducts, Bull. Belg. Math. Soc. Simon Stevin 4 (1997), no. 5, 647671.

[8] S. Dăscălescu, G. Militaru, and Ş. Raianu, Crossed coproducts and cleft coextensions, Comm. Algebra 24 (1996), no. 4, 1229-1243.

[9] S. Dăscălescu, C. Năstăsescu, and Ş. Raianu, Hopf Algebras. An Introduction, Monographs and Textbooks in Pure and Applied Mathematics, vol. 235, Marcel Dekker, New York, 2001.

[10] S. Dăscălescu, S. Raianu, and Y. H. Zhang, Finite Hopf-Galois coextensions, crossed coproducts, and duality, J. Algebra 178 (1995), no. 2, 400-413.

[11] Y. Doi and M. Takeuchi, Cleft comodule algebras for a bialgebra, Comm. Algebra 14 (1986), no. 5, 801-817.

[12] T. X. Ju and C. R. Cai, Twisted Hopf module coalgebras, Comm. Algebra 28 (2000), no. $1,307-320$.

[13] S. Montgomery, Hopf Algebras and Their Actions on Rings, CBMS Regional Conference Series in Mathematics, vol. 82, American Mathematical Society, Rhode Island, 1993.

[14] M. E. Sweedler, Cohomology of algebras over Hopf algebras, Trans. Amer. Math. Soc. 133 (1968), 205-239.

[15] _ Hopf Algebras, Mathematics Lecture Note Series, W. A. Benjamin, New York, 1969.

S. Caenepeel: Faculty of Applied Sciences, Vrije Universiteit Brussel (VUB), B-1050 Brussels, Belgium

E-mail address: scaenepe@vub.ac.be

Dingguo Wang: Department of Mathematics, Qufu Normal University, Qufu, Shandong 273165, China

E-mail address: dgwang@qtnu.edu.cn

Yanxin Wang: Department of Mathematics, Tsinghua University, Beijing 100084, China

E-mail address: wyx01@mai1s.tsinghua.edu.cn 


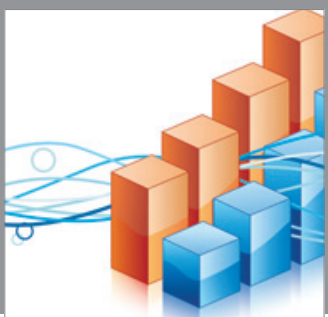

Advances in

Operations Research

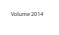

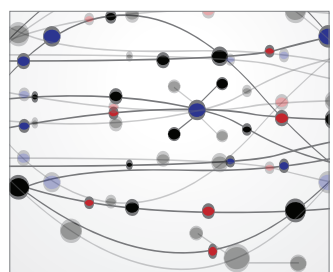

\section{The Scientific} World Journal
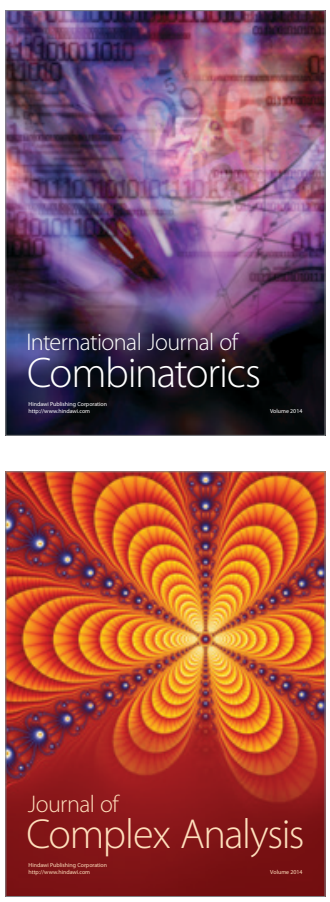

International Journal of

Mathematics and

Mathematical

Sciences
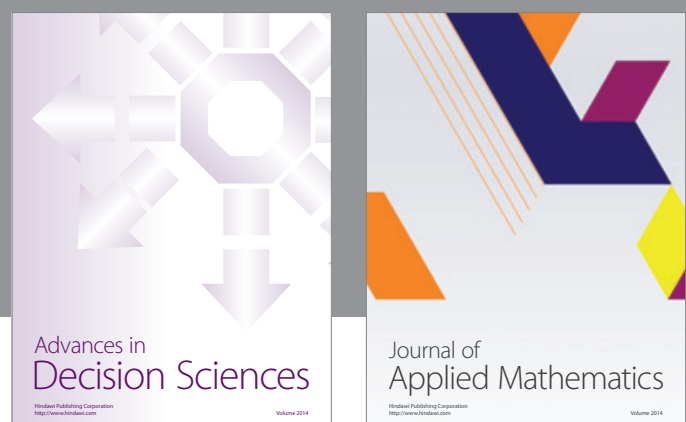

Journal of

Applied Mathematics
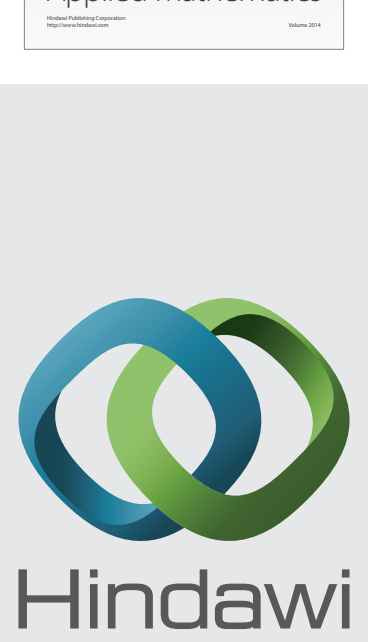

Submit your manuscripts at http://www.hindawi.com
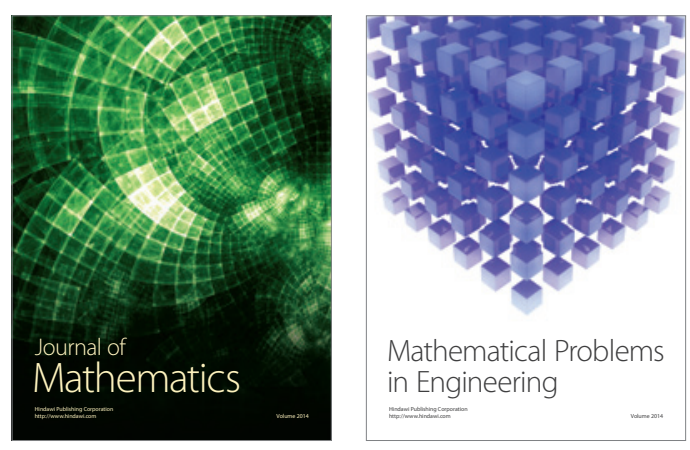

Mathematical Problems in Engineering
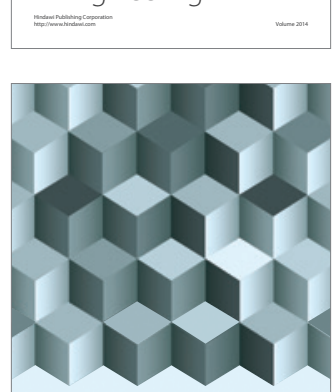

Journal of

Function Spaces
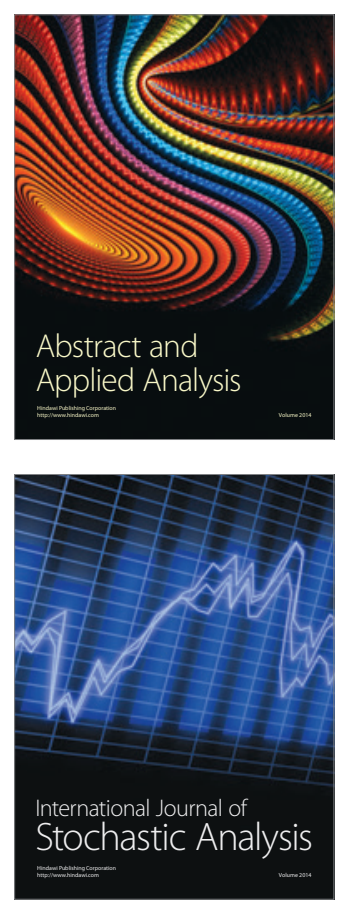

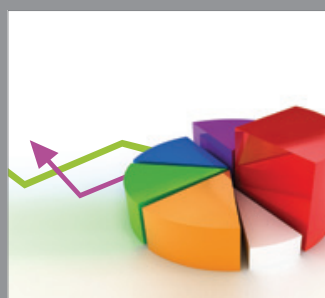

ournal of

Probability and Statistics

Promensencen
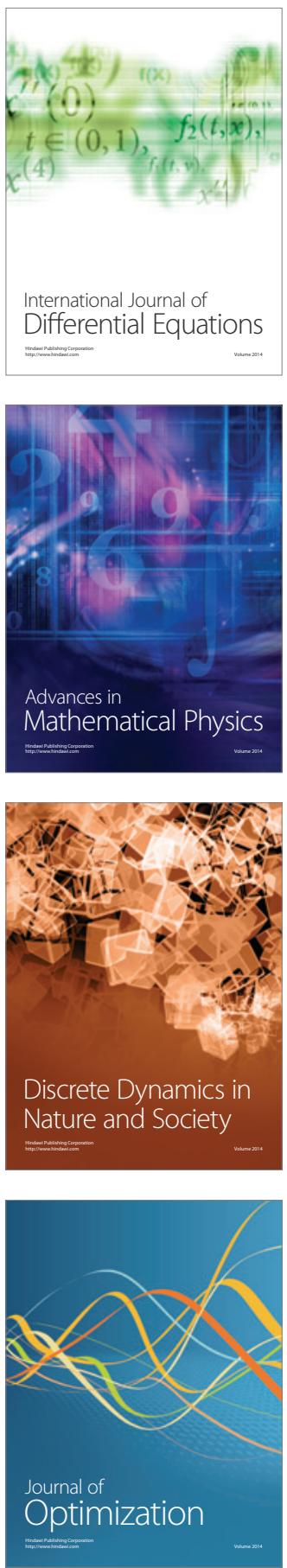